\title{
Dynamics of Equilibrium Prices With Differential and Delay Differential Equations Using Characteristic Equation Techniques
}

\author{
Martin Anokye ${ }^{1}$, Henry Amankwah ${ }^{1}$, Emmanuel Kwame Essel ${ }^{1} \&$ Irene Kafui Amponsah ${ }^{2}$ \\ ${ }^{1}$ Department of Mathematics, University of Cape Coast, Cape Coast, Ghana \\ ${ }^{2}$ Department of Statistics, University of Cape Coast, Cape Coast, Ghana \\ Correspondence: Martin Anokye, Department of Mathematics, University of Cape Coast, Cape Coast, Ghana
}

Received: April 3, 2019 Accepted: June 12, 2019 Online Published: June 26, 2019

doi:10.5539/jmr.v11n4p1 URL: https://doi.org/10.5539/jmr.v11n4p1

\begin{abstract}
This study compares differential model to delay differential model in terms of their qualitative behaviour with respect to equilibrium price changes using roots of characteristic equation techniques. The equilibrium states of both price adjustment models were simulated using inputs from same source. The study found that irrespective of initial prices set for the system, the current price of the differential model would always move monotonically towards the equilibrium price defined for the system. However, the current price of the delay- differential model will fluctuate and move away from the initial prices due to the delay parameter associated with the supply, then gradually decrease and turn towards the defined system equilibrium price.

Results from the study also showed that current prices in the delay-differential model are not predictable at the initial stage due to the time delay parameter in the supply function of price. On the other hand, current prices in their counterpart models without delay are predictable, as they always converge to the equilibrium price points defined in the system. Since most economic and physical systems are time delay inherent, it is recommended that such systems are modeled using delay-differential equations to reflect realities of the phenomena.
\end{abstract}

Keywords: delay differential equations, differential equations and equilibrium state of prices

\section{Introduction}

The behaviour of oscillation of delay differential equations and the associated differential equations without delay are not always the same in terms of their solutions (Grace and Lalli, 1985). In both theory and applications, the crucial difference is that a delay differential equation is infinite dimensional and it requires a bit more mathematical expediency to solve as compared to its ordinary differential equation counterpart (Bodnar and Piotrowska, 2010).

Time-delay inherent systems are usually modeled by delay differential equations. Such delays often appear in many control systems either in the state, the control input, or in the measurements, yet only few scientists apply these equations due to the complexity associated with their solutions. The issue of stability and the performance of time-delay control systems are therefore, both of theoretical and practical importance (Bodnar and Piotrowska, 2010; Liangliang and Jiguo, 2011; Nelson, 2006; Fridman and Uri, 2003).

Now due to the understanding that most processes involve after effect phenomena in their inner dynamics, interest related to the theory of delay differential equations has been increasing. These models are supposed to be applied in a wide variety of physical, chemical, engineering, economic, weapons fire-control from moving platforms and biological systems (Bodnar and Piotrowska, 2010; Adomian and Rach, 1983; Ligang et al, 2015).

Roots of the characteristic equation usual provide qualitative information about variables whose evolutions are described by dynamic equations. Generally, if a differential equation is parameterized on time, the variable's evolution will be stable if and only if the real part of each root is negative. For difference equations, there is stability if and only if the modulus (absolute value) of each root is less than 1. For both types of equations, persistent fluctuations occur if there is at least one pair of complex roots (Smith, 1906).

Therefore, this study seeks to use delay differential equation that is a limiting case of these equations as price adjustment model and then qualitatively compare the state of stability with its differential counterpart using characteristic equation technique. This technique is applied in the study due to complexity of solutions for systems that are linearized around equilibria, especially when the solutions involve complex variables (Smith, 1906). 
There is no doubt, that the outcomes of the study would enhance the understanding of the qualitative behavior and solutions of delay differential and differential equations so that real-life systems that are time parameterized could be modeled using appropriate mathematical approach.

\section{Methods}

This paper presents the dynamics of stability conditions of both differential and delay-differential equations applied as price adjustment functions. Therefore, the two mathematical methods are used to model time dependent processes in economics, specifically, demand and supply of commodities and then qualitatively examine their stability behaviour using characteristic equation techniques.

\subsection{Price Adjustment Differential Model Without Delay}

Given demand and supply functions of price with time equation respectively of the form

$$
\begin{gathered}
D_{p(t)}=\alpha+\beta P_{(t)} ; \quad \beta<0 \\
S_{p(t)}=\lambda+\delta P_{(t)} ; \quad \delta>0
\end{gathered}
$$

where $\alpha$ and $\lambda$ are intercepts, $\beta$ is the change in demand and $\delta$ is the change in supply.

The assumption is that the demand and supply of the commodity are dependent on only the price set for the commodity in a single market where there are no substitutes. Also, from the Walrasian model assumption, the rate of change of price involving the (1) and (2) is given by

$$
P_{(t)}^{\prime}=\gamma\left(D_{p_{(t)}}-S_{p_{(t)}}\right) .
$$

Where $\gamma>0$ is the rate of change that makes (1) and (2) results in equilibrium. Substituting (1) and (2) into (3) gives

$$
P_{(t)}^{\prime}=\gamma\left(\alpha-\lambda+(\beta-\delta) P_{(t)}\right) .
$$

Equation (4) is simplified if the following notations are adopted: let $-\rho=\gamma(\beta-\delta)>0$ and

$p_{e}=\frac{\alpha-\lambda}{\delta-\beta}>0$, then dividing through (4) by $(\delta-\beta)$, we have

$$
P_{(t)}^{\prime}=-\rho\left(P_{(t)}-P_{e}\right),
$$

where $\mathrm{P}_{e}$ is the equilibrium price. At $\mathrm{P}_{t}=\mathrm{P}_{e}$ there is no price change. If $Z_{(t)}=P_{(t)}-P_{e}$ denotes the deviation from equilibrium then $Z^{\prime}{ }_{(t)}=P^{\prime}{ }_{(t)}$ and (5) is reduced to the form

$$
Z_{(t)}^{\prime}=-\rho Z_{(t)},
$$

which could be solved by method of characteristics if $Z_{(t)}=C e^{\varphi t}$ where $\mathrm{C}$ is a constant and $\varphi$ is the root of the (6).

This implies that

$$
\varphi C e^{\varphi t}=-\rho C e^{\varphi t}
$$

and thus,

$$
(\varphi+\rho) C e^{\varphi t}=0 \Rightarrow \varphi=-\rho .
$$

Using the notation under (5), the general solution in terms of price at time $(t)$ is given by

$$
P_{(t)}=P_{e}+C e^{-\rho t} .
$$

The particular solution at time $t=0$, with initial price (condition) $P_{(0)}=P_{0}$ is also obtained as 


$$
P_{(t)}=P_{e}+\left(P_{0}-P_{e}\right) e^{-\rho t}
$$

This means that irrespective of the initial price $\mathrm{P}_{0}$ set for the (9), as $t \rightarrow \infty, e^{-\infty} \rightarrow 0$ and so $P_{(t)}=P_{e}$ after a long
period of time (Soltes et al, 2012). 2.2 Price Adjustment Differential Model With Delay

If (2) is considered to respond to price change with certain time-delay then supply functions of price with time-delay equation is given in the form

$$
S_{p(t)}=\lambda+\delta P_{(t-\tau)} ; \quad \delta>0,
$$

where $\tau$ is the time needed for change in supply, in response to a unit change in price of the commodity. Then from (3), the rate of change is mathematically expressed as follows

$$
P_{(t)}^{\prime}=\gamma\left(\alpha-\lambda+\beta P_{(t)}-\delta P_{(t-\tau)}\right) .
$$

If equation (11) is expanded and the following notations: $q=\gamma(\alpha-\lambda)$ with $\alpha>\lambda, \mathrm{r}=-\gamma \beta$ and $s=\gamma \delta$ are adopted then dividing through the resulting equation by $(\delta-\beta) \delta-\beta)$ have

$$
P^{\prime}{ }_{t}+r\left(P_{t}-P_{e}\right)+s\left(P_{t-\tau}-P_{e}\right)=0
$$

Where $p_{e}=\frac{\alpha-\lambda}{\delta-\beta}>0$, is the equilibrium price. Let $Z_{(t)}=\left(P_{(t)}-P_{e}\right)$ and $Z_{(t-\tau)}=\left(P_{(t-\tau)}-P_{e}\right)$ denote the deviations from equilibrium then at $\mathrm{P}_{t}=\mathrm{P}_{e}$ there is no change in price. It is also true that $Z_{(t)}^{\prime}=P_{(t)}^{\prime}$ and so we derive

$$
Z_{(t)}^{\prime}+r Z_{(t)}+s Z_{(t-\tau)}=0 .
$$

Equation (13) could be solved using characteristic techniques such that if $Z_{\mathrm{cs}}=C e^{\mu t} \quad$ then

$$
\mu C e^{\mu t}+r C e^{\mu t}+s C e^{\mu(t-\tau)}=0 .
$$

This implies that

$$
(\mu+r) C e^{\mu t}=-s C e^{\mu(t-\tau)}
$$

and

$$
(\mu+r)=-s e^{-\mu \tau}
$$

By setting $m=\mu+r$, we have

$$
m e^{(m-r) \tau}=-s .
$$

By multiplying through by $e^{r \tau}$, we obtain

$$
m e^{m \tau}=-s e^{r \tau}
$$

Since $s, r$ and $\tau$ are provided, the right hand side of the (15) could be represented by $\sigma$ so that

$$
F_{(m)}=m e^{m \tau}+\sigma .
$$

It is obvious that no real and positive values of $m$ satisfy this transcendental equation, however, for some range of $\sigma$ there are complex roots with positive real parts. In this case, the solution would provide oscillations that increase exponentially.

Also, if $m=x \pm i y$, and $m e^{m \tau}+\sigma=0$, then

$$
(x+i y) e^{(x+i y) \tau}+\sigma=0
$$


For which

$$
(x+i y) e^{i y \tau}=-\sigma e^{-x \tau}
$$

or

$$
(x+i y)(\cos (y \tau)+i \sin (y \tau))=-\sigma e^{-x \tau} .
$$

Then the following equations are obtained:

$$
x \cos (y \tau)-y \sin (y \tau)=-\sigma e^{-x \tau}
$$

and

$$
y \cos (y \tau)+x \sin (y \tau)=0
$$

From (18), $x=-y \cot (y \tau), \quad y \neq 0$ and we notice that

$$
\begin{aligned}
\lim _{y \rightarrow 0}-y \cot (y \tau) & =\lim _{y \rightarrow 0} \frac{-y \tau \cos (y \tau)}{\tau \sin (y \tau)} \\
& =\lim _{y \rightarrow 0}\left(\frac{-\cos (y \tau)}{\tau}\right) \lim _{y \rightarrow 0}\left(\frac{y \tau}{\sin (y \tau)}\right) \\
& =\frac{-1}{\tau} .
\end{aligned}
$$

Now, if $x \rightarrow \frac{-1}{\tau}$,as, $y \rightarrow 0$ and (17) and (18) are satisfied by $(x, y)=\left(\frac{-1}{\tau}, 0\right)$ then $\sigma=\frac{1}{\tau e}$. Therefore, a particular solution of (13) in terms of price at $t=\tau=0$ is given by

$$
P_{(t)}=P_{e}+\frac{1}{2}\left(P_{0}-P_{e}\right) e^{-\mu t}+\frac{1}{2}\left(P_{0}-P_{e}\right) e^{-\mu(t-\tau)},
$$

where $\mu=\left(\frac{1+\tau r}{\tau}\right)$ and (19) will also move to equilibrium irrespective of the initial price of the commodity. However, the movement would be controlled by the delay parameter $\tau$ in the system (Soltes et al, 2012; Falbo, 1995).

\section{Results and Discussion}

The results of the paper are now presented using same parameter values for both the differential and the delay differential price adjustment models (9) and (19) respectively, to simulate the equilibrium prices when the systems are in their stable states using MatLab.

\subsection{Equilibrium Price Set Between the Initial Prices}

The equilibrium prices are fixed at GHS 0.60 and GHS 0.65, in between the initial prices of GHS 1.70 and GHS 0.20 respectively. The price adjustment models are numerically run for both delay differential and differential systems and we had the following results.

\section{Delay-Differential Models:}

The time-delays are set at 1:00 and 0.20 at different occasions and then defined the equilibrium prices at stated earlier. The delay differential model was run numerically and following two graphical results obtained. 


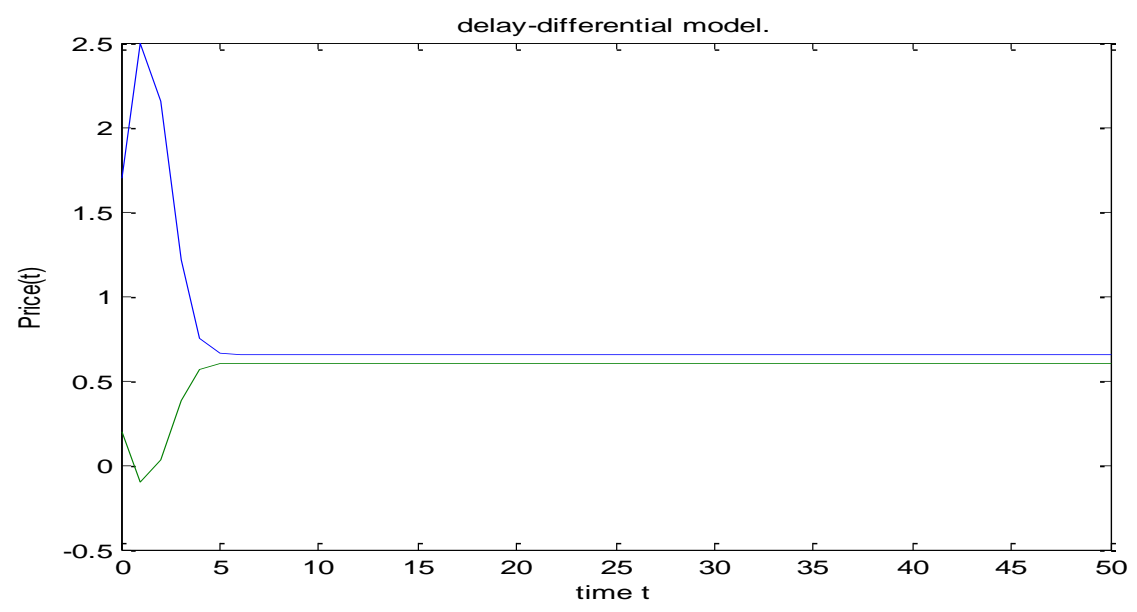

Figure 1. For delay of 1.00 and System Defined Equilibria of GHS 0.60 and GHS 0.65

In Figure 1, when the initial prices were fixed at GHS 0.20, GHS 1.70 and the equilibrium also at GHS 0.60 GHS 0.65 respectively, the current price started rising far above the initial prices due to the delay (1.00) incorporated in the model, then at point in time began decreasing to settle at equilibria defined above.

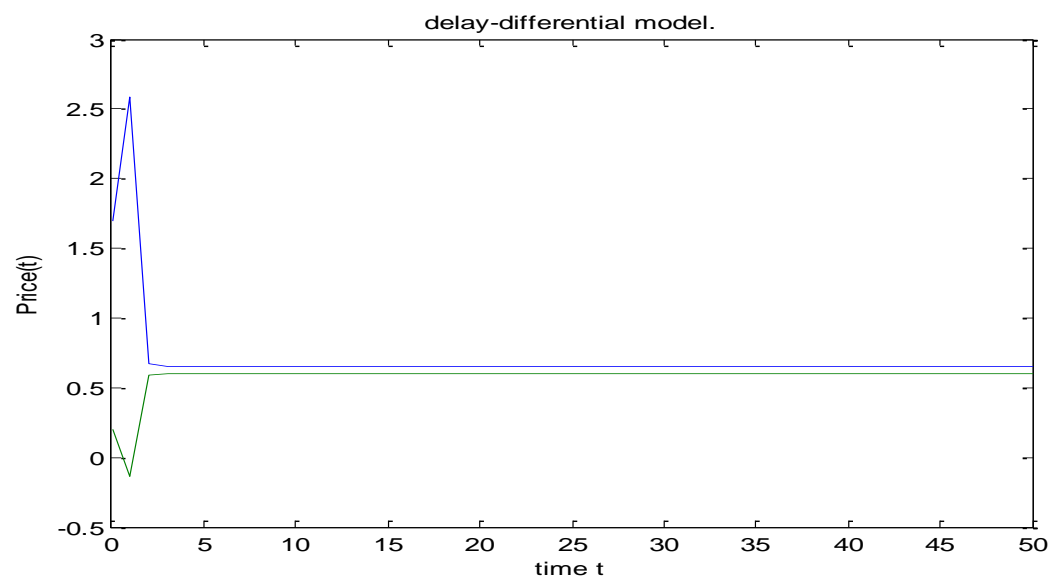

Figure 2. For a delay of 0.20 and System Defined Equilibria of GHS 0.60 and GHS 0.65

From Figure 2, the current price initiated upward movement due to the time delay value of 0.02 , then suddenly turned, decreasing towards equilibrium prices of GHS 0.65 and GHS 0.60 .

Differential Models:

The differential model was also run numerically using the system defined equilibrium prices of GHS 0.60 and GHS 0.65 and we obtained results as appeared in Figure 3. 


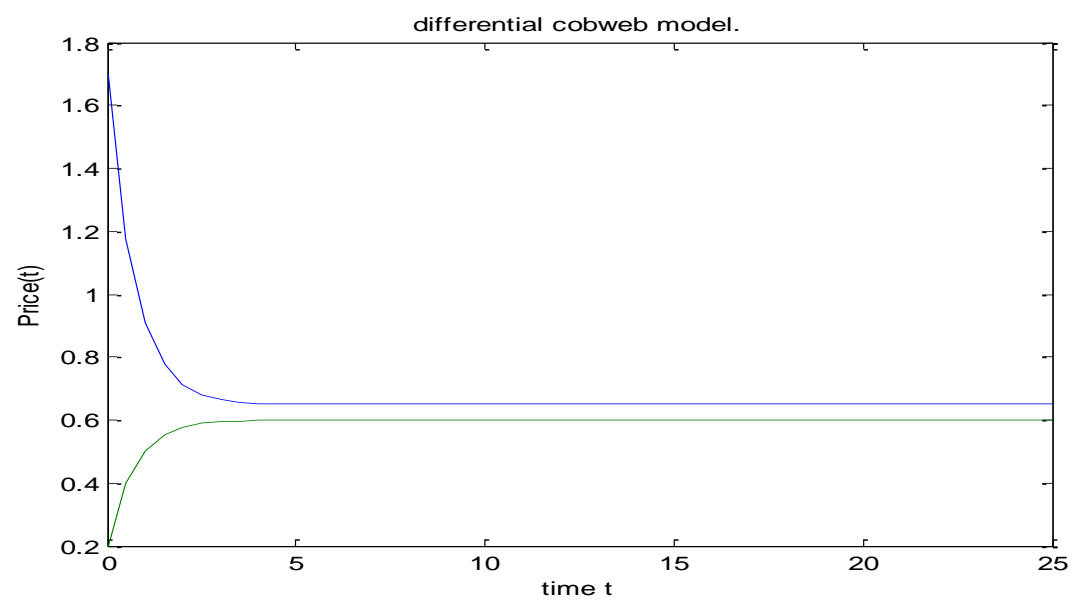

Figure 3. System Defined Equilibria of GHS 0.60 and GHS 0.65

It is observed in Figure 3, that the current price moved monotonically to the system defined equilibrium prices of GHS 0.65 and GHS 0.60 from the initial prices of GHS 1.70 and GHS 0.20 respectively.

\subsection{Equilibrium Price Set Beyond the Initial Prices}

The equilibrium price is now fixed above the initial prices at GHS 23.0, whilst the initial prices of the commodity maintained at GHS 0.20 and GHS 1.70 . The system was run numerically and the following graphical representations obtained for both delay differential and differential models.

\section{Delay-Differential Models:}

The parameter values are run for the delay-differential price adjustment model with differential time-delay values but at same system defined equilibrium prices GHS 2.30 and we had the results as shown in Figures 4 and 5.

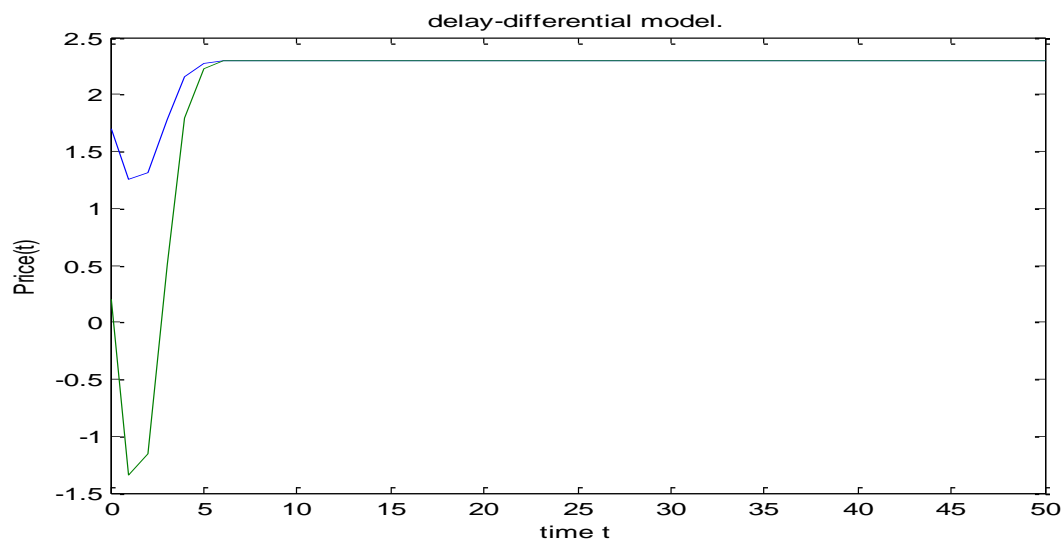

Figure 4. For a delay of 1.00 and System Defined Equilibrium of GHS 2.30

In Figure 4, when only one equilibrium price was set at GHS 2.30, above the initial prices of GHS 0.20 and GHS 1.70, the oscillatory behavoiur of the delay price function did not change because of the time-delay value of 1.00 . At different initial prices, the current price moved upwards and then with time settled at equilibrium price of GHS 2.30 defined for the system. 


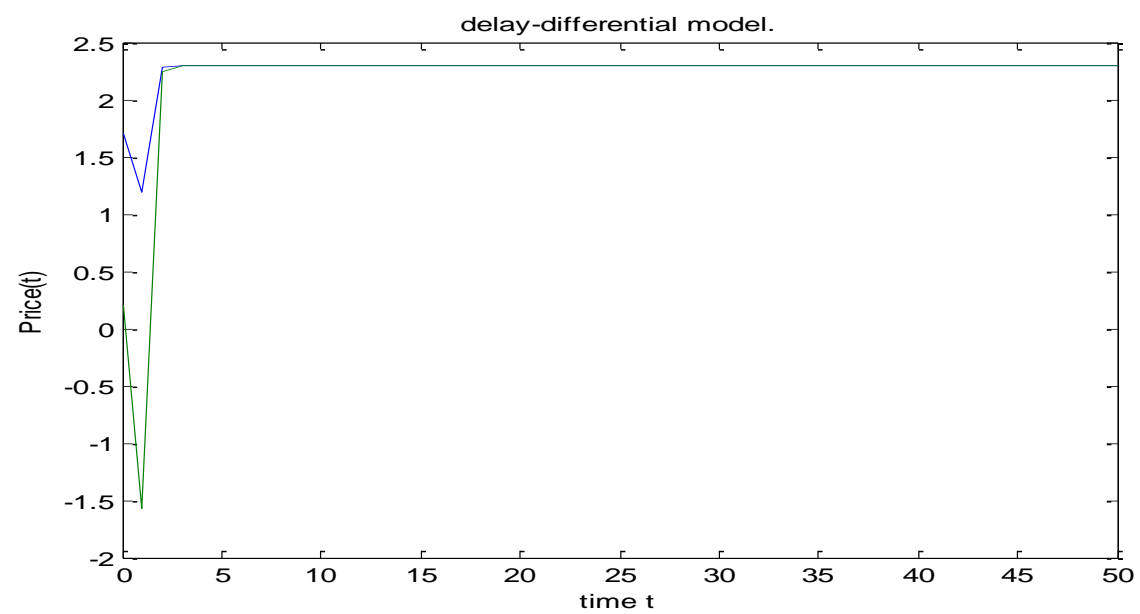

Figure 5. For a delay of 0.2 and System Defined Equilibrium of GHS 2.30

In Figure 5, it is also observed that at common defined equilibrium price of GHS 2.30 and time-delay of 0.2 , the current price converged to the equilibrium point of GHS 2.30, when the system was fixed with initial prices of GHS 0.20 and GHS1.70.

\section{Differential Models:}

For common system defined equilibrium price of GHS 2.30, the numerical results of the differential price adjustment model also provided the results as follows.

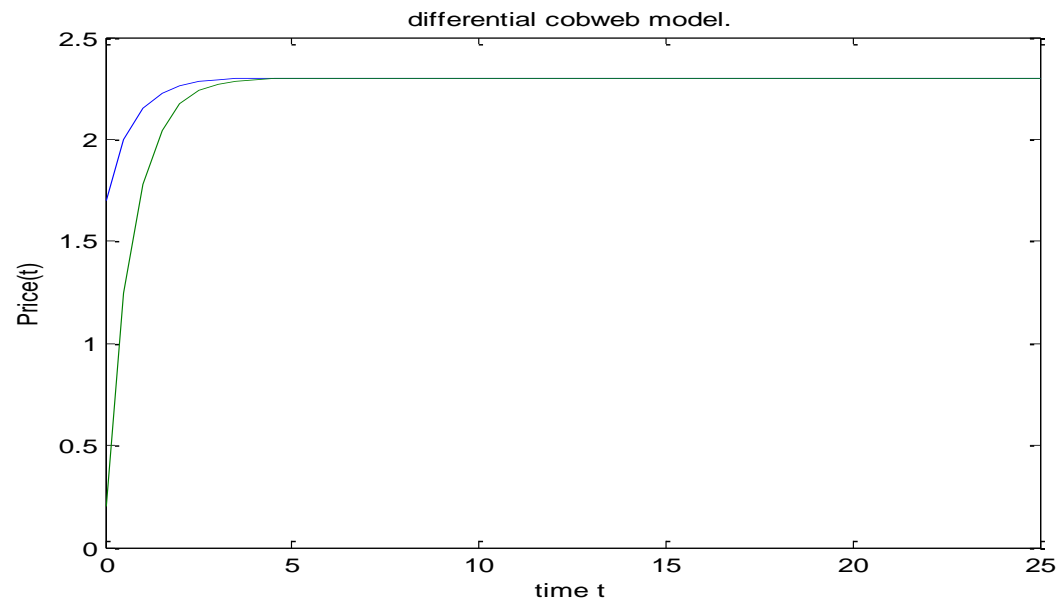

Figure 6. System Defined Equilibria of GHS 2.30

From Figure 6, it is deduced that whenever the defined equilibrium price point of differential model is set beyond the initial prices, the current price will also move towards it.

\section{Conclusion}

This paper studies the dynamics of stability states of price adjustment differential equations with and without delay parameter using characteristic equation techniques. The states of stability of the two models were simulated using their particular solutions with inputs from same source.

It was found that irrespective of initial prices set for the commodity, the current price for the differential models will always have the propensity to move monotonically to the equilibrium prices defined for the system.

On the other hand, the current price for the delay- differential models tends to oscillate and move away from the initial price due to the delay associated with the supply, then with time decreases and turns towards to the defined system equilibrium prices.

The current prices in the delay-differential models are not predictable at the initial stages due the time-delay parameters associated with them. However, the current prices of differential models without delay could be predicted as such models do not oscillate and converge monotonically to the equilibrium price points defined to be operated by the system. 
Since most economic and natural phenomena are associated with delays, it is recommended that such systems are modeled using delay-differential equations to reflect realities of the phenomena.

\section{References}

Adomian, G., \& Rach, R. (1983). Nonlinear Stochastic Differential Delay Equations, Journal of Mathematical Analysis and Applications, 91, 94-101. https://doi.org/10.1016/0022-247X(83)90094-X

Bodnar, M., \& Piotrowska, M. J. (2010). Delay differential equations: theory and applications Mathematica Applicanda (Matematyka Stosowana), 38(1), 17-56. https://doi.org/10.14708/ma.v38i1.258

Falbo, C. E. (1995). Analytic and Numerical Solutions to the Delay Differential Equation-Revised, Sonoma State University, Accessed 19 April 2018. Retrieved from www.mathfile.net/My_San_Luis_Obispo_paper.pdf

Fridman, E., \& Uri, S. (2003). Special issue on time-delay systems, Int. J. Robust Nonlinear Control, 13, 791-792. https://doi.org/10.1002/rnc.844

Grace, S. R., \& Lalli, B. S. (1985). Oscillation Theorems for Certain Delay Differential Inequalities. Journal of Mathematical Analysis and Applications, 106, 414-426. https://doi.org/10.1016/0022-247X(85)90119-2

Liangliang, W., \& Jiguo, C. (2011). Estimating Parameters in Delay Differential Equation Models. Journal of Agricultural, Biological, and Environmental Statistics, Accessed 23 April 2018, available www.stat.sfu.ca/ cao/Research/EstimateDDE.pdf

Ligang, W., Hak-Keung, L., Yuxin, Z., \& Zhan, S. (2015). Time-Delay Systems and Their Applications in Engineering, Mathematical Problems in Engineering. https://doi.org/10.1155/2015/246351

Nelson, P. (2006). Research Statement, Accessed 16 March 2018 Available www.math.lsa.umich.edu/ pwn/Research06.pdf

Smith, D. E. (1906). History of Modern Mathematics: Differential Equations. University of South Florida.

Soltes, V., Baculikova, B., \& Dzurina, J. (2012). Oscillation in Price Adjustment Models, International Journal of Business and Social Science, 3(15).

\section{Copyrights}

Copyright for this article is retained by the author(s), with first publication rights granted to the journal.

This is an open-access article distributed under the terms and conditions of the Creative Commons Attribution license (http://creativecommons.org/licenses/by/4.0/). 